\title{
Article \\ NMR-Based Lipid Metabolite Profiles to Predict Outcomes in Patients Undergoing Interventional Therapy for a Hepatocellular Carcinoma (HCC): A Substudy of the SORAMIC Trial
}

\author{
Thomas Geyer $^{1, *}$, Johannes Rübenthaler ${ }^{1}{ }^{(D)}$, Marianna Alunni-Fabbroni ${ }^{1}{ }^{(D)}$, Regina Schinner ${ }^{1}$, Sabine Weber ${ }^{2}{ }^{(D)}$, \\ Julia Mayerle ${ }^{2}$, Eric Schiffer ${ }^{3}$, Sebastian Höckner ${ }^{3}{ }^{(D}$, Peter Malfertheiner ${ }^{1,2}$ and Jens Ricke ${ }^{1}$
}

1 Department of Radiology, University Hospital, LMU Munich, Marchioninistr. 15, 81377 Munich, Germany; Johannes.ruebenthaler@med.uni-muenchen.de (J.R.); Marianna.Alunni@med.uni-muenchen.de (M.A.-F.); regina.schinner@med.uni-muenchen.de (R.S.); Peter.Malfertheiner@med.uni-muenchen.de (P.M.); Jens.Ricke@med.uni-muenchen.de (J.R.)

2 Department of Medicine II, University Hospital, LMU Munich, Marchioninistr. 15, 81377 Munich, Germany; sabine.weber@med.uni-muenchen.de (S.W.); julia.mayerle@med.uni-muenchen.de (J.M.)

3 Numares AG, Am BioPark 9, 93053 Regensburg, Germany; eric.schiffer@numares.com (E.S.); sebastian.hoeckner@numares.com (S.H.)

check for updates

Citation: Geyer, T.; Rübenthaler, J.; Alunni-Fabbroni, M.; Schinner, R.; Weber, S.; Mayerle, J.; Schiffer, E.; Höckner, S.; Malfertheiner, P.; Ricke, J. NMR-Based Lipid Metabolite Profiles to Predict Outcomes in Patients Undergoing Interventional Therapy for a Hepatocellular Carcinoma (HCC): A Substudy of the SORAMIC Trial. Cancers 2021, 13, 2787. https://doi.org/10.3390/ cancers13112787

Academic Editor: Matias A. Avila

Received: 2 May 2021

Accepted: 31 May 2021

Published: 3 June 2021

Publisher's Note: MDPI stays neutral with regard to jurisdictional claims in published maps and institutional affiliations.

Copyright: () 2021 by the authors. Licensee MDPI, Basel, Switzerland. This article is an open access article distributed under the terms and conditions of the Creative Commons Attribution (CC BY) license (https:// creativecommons.org/licenses/by/ $4.0 /)$.
* Correspondence: thomas.geyer@med.uni-muenchen.de; Tel.: +49-89-4400-73620

Simple Summary: A hepatocellular carcinoma (HCC) is the most common cause of death in patients suffering from chronic liver diseases. In order to improve the prediction of outcomes in HCC patients, there is a need for new biomarkers. This pilot study aimed at identifying serum metabolites for the prediction of outcomes of HCC patients using nuclear magnetic resonance (NMR) spectroscopy. This analysis revealed that high serum concentrations of myo-inositol or dimethylamine were associated with an improved overall survival. In contrast, high concentrations of total cholesterol, LDL-cholesterol and LDL particles (LDL-P) were associated with a decreased overall survival. The identification of novel biomarkers using this NMR-based technology holds promise for opening new directions in the conduction of interventional trials in HCCs.

Abstract: Background: This exploratory study aimed to evaluate lipidomic and metabolomic profiles in patients with early and advanced HCCs and to investigate whether certain metabolic parameters may predict the overall survival in these patients. Methods: A total of 60 patients from the prospective, randomized-controlled, multicenter phase II SORAMIC trial were included in this substudy; among them were 30 patients with an early HCC who underwent radiofrequency ablation combined with sorafenib or a placebo and 30 patients with an advanced HCC who were treated with a selective internal radiation therapy (SIRT) plus sorafenib vs. sorafenib alone. The blood serum of these patients was analyzed using a standardized nuclear magnetic resonance (NMR) platform. All tested metabolites were correlated with the overall survival. Results: The overall survival (OS) was significantly higher in patients with an early HCC (median OS: 34.0 months) compared with patients with an advanced HCC (median OS: 12.0 months) $(p<0.0001)$. Patients with high serum concentrations of myo-inositol (MI) had a higher overall survival compared with patients with low concentrations (21.6 vs. 13.8 months) with a Pearson correlation coefficient of 0.331 ( $p=0.011$ ). Patients with high serum concentrations of dimethylamine had a higher overall survival compared with patients with low concentrations (25.1 vs. 19.7 months) with a Pearson correlation coefficient of $0.279(p=0.034)$. High concentrations of total cholesterol, LDL-cholesterol and LDL particles (LDL-P) were associated with a decreased overall survival. Conclusions: NMR-based lipidomic and metabolomic profiling has the potential to identify individual metabolite biomarkers that predict the outcome of patients with an HCC exposed to non-invasive therapeutic management.

Keywords: HCC; overall survival; metabolomics; NMR; lipid profiles; interventional therapy; SIRT; radiofrequency ablation 


\section{Introduction}

In order to improve and personalize the prediction of the overall survival in HCC patients, there is a need for new and accurate biomarkers. Due to the essential metabolic functions of the liver, an HCC is an ideal model for metabolomics research with encouraging insights into the pathogenesis and a possible translation for clinical applications [1-5]. Metabolic alterations are well established in an HCC with distinct lipid accumulations depending on the degree of the tumor differentiation [6]. Metabolomics technologies, therefore, have gained attention as they may lead to the detection of novel biomarkers for the diagnosis and monitoring of liver cancer and various other diseases [3,7]. This technology allows the identification of small molecule metabolic profiles of complex biological matrices and the evaluation of metabolites generated in response to several exogenous and endogenous factors [8]. The two main platforms for analyses in metabolomics are nuclear magnetic resonance (NMR) spectroscopy and mass spectrometry (MS) [3]. Both techniques allow a quantitative analysis of multiple metabolites in a single analytical step. Although mass spectrometry-based approaches can resolve a higher number of metabolites than NMR spectroscopy, clear advantages of the NMR technique include the non-destructive character of the analysis, simple sample processing and high analytical reproducibility. A standardized NMR platform was shown to be a powerful analytical tool for an in-depth lipoprotein analysis in various contexts [9-13]. Recently, a metabolomic analysis using the same technology and AI-based approaches allowed the identification of constellations of urinary and blood metabolites as novel biomarkers for a non-invasive assessment of a renal allograft rejection $[14,15]$ and the precise estimation of a kidney function [16], respectively.

The present exploratory study aimed to evaluate NMR-based lipidomic and metabolomic profiles in patients with early and advanced HCCs using NMR spectroscopy and to investigate whether certain metabolic parameters/profiles may predict outcomes in these patients.

\section{Materials and Methods}

\subsection{Study Design}

The present exploratory study is a substudy of the prospective, randomized-controlled, multicenter phase II SORAMIC trial (EudraCT 2009-012576-27, NCT01126645), which was conducted in 12 countries in Europe and Turkey $[17,18]$. The study was approved by the institutional review boards of all 38 participating centers and conducted according to the ethical principles expressed in the Declaration of Helsinki. Written informed consent was obtained from all participants. The SORAMIC trial comprised of a diagnostic, a curative and a palliative part.

A total of 60 patients were included in this study (Figure 1) and consisted of the following subgroups:

- A total of 30 patients (24 male, 6 female; mean age 67, range: 53-83) with an early HCC (BCLC A) and liver cirrhosis who underwent a local ablation within the SORAMIC trial (radiofrequency ablation combined with sorafenib or a placebo)

- A total of 30 patients (28 male, 2 female; mean age 66, range: 41-79) with an advanced HCC (BCLC B or C) and liver cirrhosis who underwent palliative treatment within the SORAMIC trial (selective internal radiation therapy (SIRT) with yttrium-90 $\left({ }^{90} \mathrm{Y}\right.$ ) resin microspheres plus sorafenib vs. sorafenib alone). 


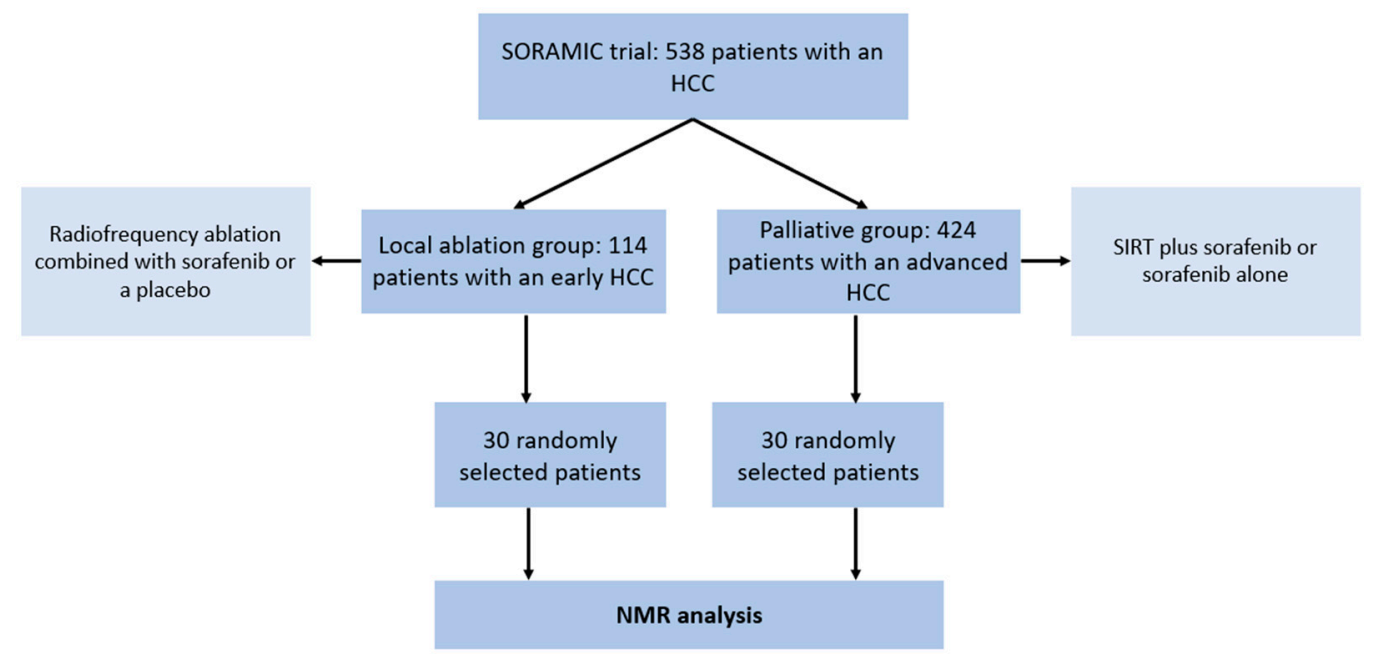

Figure 1. Flowchart illustrating the patient cohorts in the SORAMIC trial and the selection of patients for the present study. HCC = hepatocellular carcinoma. NMR = nuclear magnetic resonance. SIRT $=$ selective internal radiation therapy.

Peripheral blood $(5 \mathrm{~mL}$ ) was drawn in S-Monovette serum tubes (Sarstedt AG, Nümbrecht, Germany) and processed immediately (centrifugation $3000 \mathrm{rpm}, 5 \mathrm{~min}, 4^{\circ} \mathrm{C}$ ) to collect serum, which was aliquoted and stored at $-80{ }^{\circ} \mathrm{C}$ until further use. The serum samples were sent to Numares AG (Regensburg, Germany) for a targeted metabolomic analysis using the standardized NMR AXINON ${ }^{\circledR}$ platform.

\subsection{NMR AXINON ${ }^{\circledR}$ Platform}

The serum was thawed at room temperature and prepared for NMR analysis using the AXINON ${ }^{\circledR}$ platform (Numares AG, Regensburg, Germany). All tested parameters are displayed in Table 1 . To this end, $630 \mu \mathrm{L}$ serum of each sample was mixed with $70 \mu \mathrm{L}$ of the AXINON ${ }^{\circledR}$ serum additive solution and a total of $600 \mu \mathrm{L}$ was transferred to $5 \mathrm{~mm}$ NMR tubes. If necessary, multiple aliquots of the same serum sample were pooled to reach the required minimum sample volume. The samples were run in several batches, each including calibration and two process control samples and were kept at $6{ }^{\circ} \mathrm{C}$ in the SampleJet auto-feeder until measurement on a Bruker Avance III $600 \mathrm{MHz}$ spectrometer equipped with a $5 \mathrm{~mm}$ PATXI probe and automatic $Z$ gradients shimming (Bruker Corporation, Billerica, MA, USA). The NMR measurement was carried out at $37^{\circ} \mathrm{C}$ after pre-heating of samples at $37^{\circ} \mathrm{C}$ for $7.5 \mathrm{~min}$. The acquisition and processing of NMR spectra and the quantification of NMR signals for a lipoprotein subclass and a small metabolite analysis were carried out as described $[9,16]$. 
Table 1. Metabolite and lipid parameters that were analyzed in the present study using the standardized $A X I N O N^{\circledR}$ platform (Numares AG, Regensburg, Germany).

\begin{tabular}{|c|c|c|}
\hline Parameter Name & Description & Unit \\
\hline Creatine & Concentration of serum creatine & $\mu \mathrm{mol} / \mathrm{L}$ \\
\hline Creatinine & Concentration of serum creatinine & $\mu \mathrm{mol} / \mathrm{L}$ \\
\hline Dimethylamine & Concentration of serum dimethylamine & $\mu \mathrm{mol} / \mathrm{L}$ \\
\hline Dimethylsulfone & Concentration of serum dimethyl sulfone & $\mu \mathrm{mol} / \mathrm{L}$ \\
\hline Glycerol & Concentration of serum glycerol & $\mu \mathrm{mol} / \mathrm{L}$ \\
\hline Isoleucine & Concentration of serum isoleucine & $\mu \mathrm{mol} / \mathrm{L}$ \\
\hline Myo-Inositol & Concentration of serum myo-inositol & $\mu \mathrm{mol} / \mathrm{L}$ \\
\hline Valine & Concentration of serum valine & $\mu \mathrm{mol} / \mathrm{L}$ \\
\hline GFR(NMR) & Glomerular filtration rate estimated from metabolite constellation & $\mathrm{mL} / \mathrm{min} / 1.73 \mathrm{~m}^{2}$ \\
\hline LVLDL-P & Concentration of large VLDL particles & $\mathrm{nmol} / \mathrm{L}$ \\
\hline LDL-P & Concentration of LDL particles & $\mathrm{nmol} / \mathrm{L}$ \\
\hline LLDL-P & Concentration of large LDL particles & $\mathrm{nmol} / \mathrm{L}$ \\
\hline SLDL-P & Concentration of small LDL particles & $\mathrm{nmol} / \mathrm{L}$ \\
\hline HDL-P & Concentration of HDL particles & $\mathrm{nmol} / \mathrm{L}$ \\
\hline LHDL-P & Concentration of large HDL particles & $\mathrm{nmol} / \mathrm{L}$ \\
\hline SHDL-P & Concentration of small HDL particles & $\mathrm{nmol} / \mathrm{L}$ \\
\hline VLDL-s & Mean diameter of VLDL particles & $\mathrm{nm}$ \\
\hline LDL-s & Mean diameter of LDL particles & $\mathrm{nm}$ \\
\hline HDL-s & Mean diameter of HDL particles & $\mathrm{nm}$ \\
\hline VLDL-c & Cholesterol concentration in VLDL class & $\mathrm{mg} / \mathrm{dL}$ \\
\hline IDL-c & Cholesterol concentration in IDL class & $\mathrm{mg} / \mathrm{dL}$ \\
\hline LDL-c & Cholesterol concentration in LDL class & $\mathrm{mg} / \mathrm{dL}$ \\
\hline LDL.A-c & Cholesterol concentration in LDL subclass A (large particles) & $\mathrm{mg} / \mathrm{dL}$ \\
\hline LDL.B-c & Cholesterol concentration in LDL subclass B (medium-sized particles) & $\mathrm{mg} / \mathrm{dL}$ \\
\hline LDL.C-c & Cholesterol concentration in LDL subclass C (small particles) & $\mathrm{mg} / \mathrm{dL}$ \\
\hline HDL.A-c & Cholesterol concentration in HDL subclass A (large particles) & $\mathrm{mg} / \mathrm{dL}$ \\
\hline HDL.B-c & Cholesterol concentration in HDL subclass B (medium-sized particles) & $\mathrm{mg} / \mathrm{dL}$ \\
\hline HDL.C-c & Cholesterol concentration in HDL subclass C (small particles) & $\mathrm{mg} / \mathrm{dL}$ \\
\hline Total-Cholesterol & Concentration of total cholesterol in serum & $\mathrm{mg} / \mathrm{dL}$ \\
\hline LDL-Cholesterol & Concentration of LDL-cholesterol in serum & $\mathrm{mg} / \mathrm{dL}$ \\
\hline HDL-Cholesterol & Concentration of HDL-cholesterol in serum & $\mathrm{mg} / \mathrm{dL}$ \\
\hline Triglycerides & Concentration of total triglycerides in serum & $\mathrm{mg} / \mathrm{dL}$ \\
\hline Alanine & Concentration of alanine in serum & $\mu \mathrm{mol} / \mathrm{L}$ \\
\hline Leucine & Concentration of leucine in serum & $\mu \mathrm{mol} / \mathrm{L}$ \\
\hline
\end{tabular}

\subsection{Statistical Analysis}

All statistical calculations were performed using SAS Version 9.4 for Windows (Copyright SAS Institute Inc., Cary, NC, USA). The serum concentrations in different subgroups were compared using an ANOVA and a t-test. For the correlation analysis, Pearson and Spearman correlation coefficients were calculated. The overall survival was calculated by the Kaplan-Meier method and the log-rank test was used to compare the survival curves. The cut-off points for distinguishing between the high and low concentration values for overall survival $</>12$ months were determined by the Youden index. Additionally, for a multivariate analysis of influence factors on the overall survival, a Cox proportional hazards model was used. The level of significance was set at alpha $\leq 0.05$.

\section{Results}

\subsection{Patient Characteristics and Treatment Groups}

A total of 60 patients with a mean age of 66 (range: 41-83) and a male predominance (52 men, 8 women; ratio: 6.5:1) was included in this study. The two subgroups consisted of patients with an early HCC $(n=30)$ and an advanced HCC $(n=30)$. The characteristics of all included patients are illustrated in Table 2; the treatment groups are displayed in Table 3. 
Table 2. Baseline characteristics of all included patients with an advanced HCC $(n=30)$ and an early HCC $(n=30)$. The mean values and standard deviations (SD, in parentheses) as well as $p$-values are displayed. ALBI = albumin-bilirubin; $\mathrm{BCLC}=$ Barcelona Clinic liver cancer staging classification; $\mathrm{BMI}=$ body mass index; $\mathrm{HBV}=$ hepatitis $\mathrm{B}$ virus; $\mathrm{HCC}=$ hepatocellular carcinoma; $\mathrm{HCV}=$ hepatitis $\mathrm{C}$ virus; $\mathrm{IQR}=$ interquartile range; $\mathrm{PVI}=$ portal vein infiltration; $\mathrm{SD}=$ standard deviation .

\begin{tabular}{|c|c|c|c|c|}
\hline \multirow[t]{2}{*}{ Variables } & \multirow{2}{*}{$\begin{array}{l}\text { Total } \\
(n=60)\end{array}$} & \multirow{2}{*}{$\begin{array}{l}\text { Early HCC } \\
(n=30)\end{array}$} & \multirow{2}{*}{$\begin{array}{l}\text { Advanced } \\
\text { HCC } \\
(n=30)\end{array}$} & \multirow{2}{*}{$p$-Value } \\
\hline & & & & \\
\hline Gender & & & & 0.1287 \\
\hline Male & $52(86.7)$ & $24(80.0)$ & $28(93.3)$ & \\
\hline Female & $8(13.3)$ & $6(20.0)$ & $2(6.7)$ & \\
\hline Age & & & & 0.6095 \\
\hline Mean (SD) & $66.4(8.2)$ & $67.0(8.2)$ & $65.9(8.4)$ & \\
\hline Median (IQR) & $66.5(12.5)$ & $65.5(12.0)$ & $67.5(14.0)$ & \\
\hline Min-Max & $41.0-83.0$ & $53.0-83.0$ & $41.0-79.0$ & \\
\hline Age & & & & 1.0000 \\
\hline$<65$ & $22(36.7)$ & $11(36.7)$ & $11(36.7)$ & \\
\hline$\geq 65$ & $38(63.3)$ & $19(63.3)$ & $19(63.3)$ & \\
\hline BMI & & & & 0.6930 \\
\hline Mean (SD) & $27.9(4.1)$ & $27.7(3.2)$ & $28.1(4.8)$ & \\
\hline Median (IQR) & $27.2(4.8)$ & $27.1(4.2)$ & $27.3(5.1)$ & \\
\hline Min-Max & $19.5-38.0$ & $22.8-35.3$ & $19.5-38.0$ & \\
\hline BMI & & & & 0.8752 \\
\hline Normal & $15(25.0)$ & $7(23.3)$ & $8(26.7)$ & \\
\hline Overweight & $30(50.0)$ & $16(53.3)$ & $14(46.7)$ & \\
\hline Obese & $15(25.0)$ & $7(23.3)$ & $8(26.7)$ & \\
\hline Etiology: HBV & & & & 0.1611 \\
\hline Yes & $5(8.3)$ & $1(3.3)$ & $4(13.3)$ & \\
\hline No & $55(91.7)$ & $29(96.7)$ & $26(86.7)$ & \\
\hline Etiology: HCV & & & & 1.0000 \\
\hline Yes & $14(23.3)$ & $7(23.3)$ & $7(23.3)$ & \\
\hline No & $46(76.7)$ & $23(76.7)$ & $23(76.7)$ & \\
\hline Etiology: Alcohol & & & & 0.1213 \\
\hline Yes & $30(50.0)$ & $18(60.0)$ & $12(40.0)$ & \\
\hline No & $30(50.0)$ & $12(40.0)$ & $18(60.0)$ & \\
\hline Child Pugh & & & & 0.7386 \\
\hline A & $49(81.7)$ & $25(83.3)$ & $24(80.0)$ & \\
\hline B & $11(18.3)$ & $5(16.7)$ & $6(20.0)$ & \\
\hline BCLC & & & & $<0.0001$ \\
\hline A & $30(50.0)$ & $30(100.0)$ & & \\
\hline B & $8(13.3)$ & & $8(26.7)$ & \\
\hline $\mathrm{C}$ & $22(36.7)$ & & $22(73.3)$ & \\
\hline Liver Dominant Disease & & & & 1.0000 \\
\hline Yes & $58(96.7)$ & $29(96.7)$ & $29(96.7)$ & \\
\hline No & $2(3.3)$ & $1(3.3)$ & $1(3.3)$ & \\
\hline Extrahepatic Metastases & & & & 0.0049 \\
\hline Yes & $7(11.7)$ & & $7(23.3)$ & \\
\hline No & $53(88.3)$ & $30(100)$ & $23(76.7)$ & \\
\hline Number of Liver Lesions & & & & $<0.0001$ \\
\hline 1 & $30(50.0)$ & $26(86.7)$ & 4 (13.3) & \\
\hline 2 & $8(13.3)$ & $4(13.3)$ & $4(13.3)$ & \\
\hline $3-10$ & $8(13.3)$ & & $8(26.7)$ & \\
\hline Diffuse Disease & $14(23.3)$ & & $14(46.7)$ & \\
\hline
\end{tabular}


Table 2. Cont.

\begin{tabular}{|c|c|c|c|c|}
\hline \multirow[t]{2}{*}{ Variables } & \multirow{2}{*}{$\begin{array}{l}\text { Total } \\
(n=60)\end{array}$} & \multirow{2}{*}{$\begin{array}{l}\text { Early HCC } \\
(n=30)\end{array}$} & \multirow{2}{*}{$\begin{array}{l}\text { Advanced } \\
\text { HCC } \\
(n=30)\end{array}$} & \multirow{2}{*}{$p$-Value } \\
\hline & & & & \\
\hline PVI & & & & 0.0015 \\
\hline Missing & $3(5.0)$ & $2(6.7)$ & $1(3.3)$ & \\
\hline Yes & $12(21.1)$ & $1(3.6)$ & $11(37.9)$ & \\
\hline No & $45(78.9)$ & $27(96.4)$ & $18(62.1)$ & \\
\hline Bilirubin (mg/dL) & & & & 0.0234 \\
\hline Mean (SD) & $16.1(7.8)$ & $18.3(8.1)$ & $13.8(7.0)$ & \\
\hline Median (IQR) & $14.7(11.0)$ & $17.4(10.4)$ & $11.6(8.6)$ & \\
\hline Min-Max & $6.0-36.4$ & $7.4-36.4$ & $6.0-35.0$ & \\
\hline Albumin (g/dL) & & & & 0.0966 \\
\hline Mean (SD) & $37.0(9.6)$ & $39.1(5.3)$ & $34.9(12.3)$ & \\
\hline Median (IQR) & $39.6(9.5)$ & $39.7(7.5)$ & $39.2(11.4)$ & \\
\hline Min-Max & $0.5-47.0$ & $27.4-47.0$ & $0.5-46.9$ & \\
\hline ALBI Score & & & & 0.6821 \\
\hline Mean (SD) & $-2.5(0.6)$ & $-2.5(0.5)$ & $-2.4(0.7)$ & \\
\hline Median (IQR) & $-2.6(0.9)$ & $-2.5(0.7)$ & $-2.6(1.2)$ & \\
\hline Min-Max & $-3.3-0.2$ & $-3.2-1.3$ & $-3.3-0.2$ & \\
\hline ALBI Grade & & & & 1.0000 \\
\hline Mean (SD) & $28(46.7)$ & $14(46.7)$ & $14(46.7)$ & \\
\hline Median (IQR) & $30(50.0)$ & $15(50.0)$ & $15(50.0)$ & \\
\hline Min-Max & $2(3.3)$ & $1(3.3)$ & $1(3.3)$ & \\
\hline
\end{tabular}

Table 3. Received treatments in patients with an early HCC and an advanced HCC within the SORAMIC trial. HCC = hepatocellular carcinoma; RFA = radiofrequency ablation; SIRT = selective internal radiation therapy.

\begin{tabular}{|c|c|c|c|c|}
\hline Group & Intention to Treat (ITT) & Actually Received Treatment & $n$ & $\begin{array}{c}\text { Treatment Group as } \\
\text { Displayed in Manuscript }\end{array}$ \\
\hline Early HCC & RFA + sorafenib & RFA + sorafenib & 15 & $\begin{array}{l}\text { Early HCC with RFA and } \\
\text { sorafenib }\end{array}$ \\
\hline Early HCC & RFA + sorafenib & RFA (no sorafenib received) & 2 & Early HCC with RFA but no \\
\hline Early HCC & RFA + placebo & RFA + placebo & 13 & sorafenib \\
\hline Advanced HCC & SIRT/sorafenib & SIRT/sorafenib & 12 & $\begin{array}{l}\text { Advanced HCC with SIRT and } \\
\text { sorafenib }\end{array}$ \\
\hline Advanced HCC & SIRT/sorafenib & Sorafenib (no SIRT received) & 2 & \\
\hline Advanced HCC & Sorafenib & Sorafenib & 14 & Advanced HCC without SIRT \\
\hline Advanced HCC & SIRT/sorafenib & (No study treatment) & 2 & \\
\hline
\end{tabular}

\subsection{NMR Data}

The serum concentration results of all parameters are shown in Table 4. Significant differences in patients with an early HCC compared with patients with an advanced HCC were detected in the cholesterol concentration in LDL subclass A (large particles) (higher in an advanced HCC) $(p=0.045)$ and in the serum concentration of lactate (higher in an early HCC) $(p=0.001)$.No significant differences in patients with an early vs. an advanced HCC were detected. 
Table 4. Serum concentration levels of the metabolite and lipid parameters that were analyzed in the present study using the standardized AXINON ${ }^{\circledR}$ platform (Numares AG, Regensburg, Germany). The mean values and standard deviations (SD, in parentheses) as well as $p$-values are displayed.

\begin{tabular}{|c|c|c|c|}
\hline Variables & $\begin{array}{c}\text { Early HCC } \\
(n=30)\end{array}$ & $\begin{array}{l}\text { Advanced HCC } \\
\quad(n=30)\end{array}$ & $p$-Value \\
\hline Creatine $(\mu \mathrm{mol} / \mathrm{L})$ & $32.2(24.4)$ & $33.0(25.2)$ & 0.9003 \\
\hline Creatinine $(\mu \mathrm{mol} / \mathrm{L})$ & $95.8(28.6)$ & $95.1(32.4)$ & 0.9396 \\
\hline Dimethylamine $(\mu \mathrm{mol} / \mathrm{L})$ & $4.3(0.6)$ & $4.4(0.7)$ & 0.4523 \\
\hline Dimethylsulfone ( $\mu \mathrm{mol} / \mathrm{L})$ & $12.2(5.1)$ & $12.1(6.4)$ & 0.9339 \\
\hline Glycerol ( $\mu \mathrm{mol} / \mathrm{L})$ & $173.0(65.4)$ & $183.6(58.8)$ & 0.5181 \\
\hline Isoleucine ( $\mu \mathrm{mol} / \mathrm{L})$ & $83.2(21.5)$ & $85.5(16.7)$ & 0.6527 \\
\hline Myo-Inositol ( $\mu \mathrm{mol} / \mathrm{L})$ & $71.3(28.8)$ & $69.1(17.3)$ & 0.7285 \\
\hline Valine $(\mu \mathrm{mol} / \mathrm{L})$ & $282.5(72.6)$ & $287.0(54.4)$ & 0.7914 \\
\hline GFR(NMR) $\left(\mathrm{mL} / \mathrm{min} / 1.73 \mathrm{~m}^{2}\right)$ & $76.7(21.4)$ & $79.8(19.6)$ & 0.5711 \\
\hline LVLDL-P (nmol/L) & $3.7(2.9)$ & $3.0(2.2)$ & 0.3076 \\
\hline LDL-P (nmol/L) & $1132.1(477.7)$ & $1291.1(586.4)$ & 0.2575 \\
\hline LLDL-P (nmol/L) & $632.8(215.0)$ & $713.8(300.7)$ & 0.2377 \\
\hline SLDL-P (nmol/L) & $506.4(343.5)$ & $581.9(329.2)$ & 0.3922 \\
\hline HDL-P (nmol/L) & 20,798.6 (7999.7) & 21,304.6 (8719.5) & 0.8171 \\
\hline LHDL-P (nmol/L) & $6039.9(2806.9)$ & $5140.3(2189.9)$ & 0.1763 \\
\hline SHDL-P (nmol/L) & $15,910.8(7652.0)$ & $17,444.8(7993.8)$ & 0.4663 \\
\hline VLDL-s (nm) & $50.5(4.8)$ & $49.7(3.5)$ & 0.4531 \\
\hline LDL-s (nm) & $21.3(0.4)$ & $21.5(0.5)$ & 0.1125 \\
\hline HDL-s (nm) & $9.3(0.5)$ & $9.2(0.4)$ & 0.2851 \\
\hline VLDL-c (mg/dL) & $24.2(7.4)$ & $25.2(10.4)$ & 0.6794 \\
\hline IDL-c (mg/dL) & $43.8(14.6)$ & $47.9(17.4)$ & 0.3253 \\
\hline LDL-c (mg/dL) & $97.3(35.3)$ & $109.8(38.8)$ & 0.2143 \\
\hline LDL.A-c (mg/dL) & $32.3(10.9)$ & $37.9(9.7)$ & 0.045 \\
\hline LDL.B-c (mg/dL) & $14.9(10.5)$ & $20.4(12.2)$ & 0.0767 \\
\hline LDL.C-c(mg/dL) & $4.1(2.1)$ & $4.0(1.8)$ & 0.8177 \\
\hline HDL.A-c (mg/dL) & $17.5(6.1)$ & $18.5(4.4)$ & 0.5037 \\
\hline HDL.B-c (mg/dL) & $15.4(2.3)$ & $15.2(1.8)$ & 0.6692 \\
\hline HDL.C-c (mg/dL) & $9.2(5.9)$ & $10.6(6.7)$ & 0.4451 \\
\hline Total-Cholesterol (mg/dL) & $159.0(53.8)$ & $181.1(47.2)$ & 0.0997 \\
\hline LDL-Cholesterol (mg/dL) & $95.1(47.1)$ & $116.2(44.3)$ & 0.0807 \\
\hline HDL-Cholesterol (mg/dL) & $42.1(11.5)$ & $42.0(10.8)$ & 0.9912 \\
\hline Triglycerides (mg/dL) & $124.4(53.0)$ & $117.2(54.6)$ & 0.6096 \\
\hline Alanine $(\mu \mathrm{mol} / \mathrm{L})$ & $508.4(112.1)$ & $471.8(93.1)$ & 0.1789 \\
\hline Leucine $(\mu \mathrm{mol} / \mathrm{L})$ & $158.4(44.0)$ & $150.7(36.2)$ & 0.4832 \\
\hline
\end{tabular}




\subsection{Overall Survival}

The overall survival (OS) was significantly longer in patients with an early HCC (median OS: 34.0 months) compared with patients with an advanced HCC (median OS: 12.0 months) $(p<0.0001)$. Kaplan-Meier curves for patients with an early vs. an advanced HCC are displayed in Figure 2.

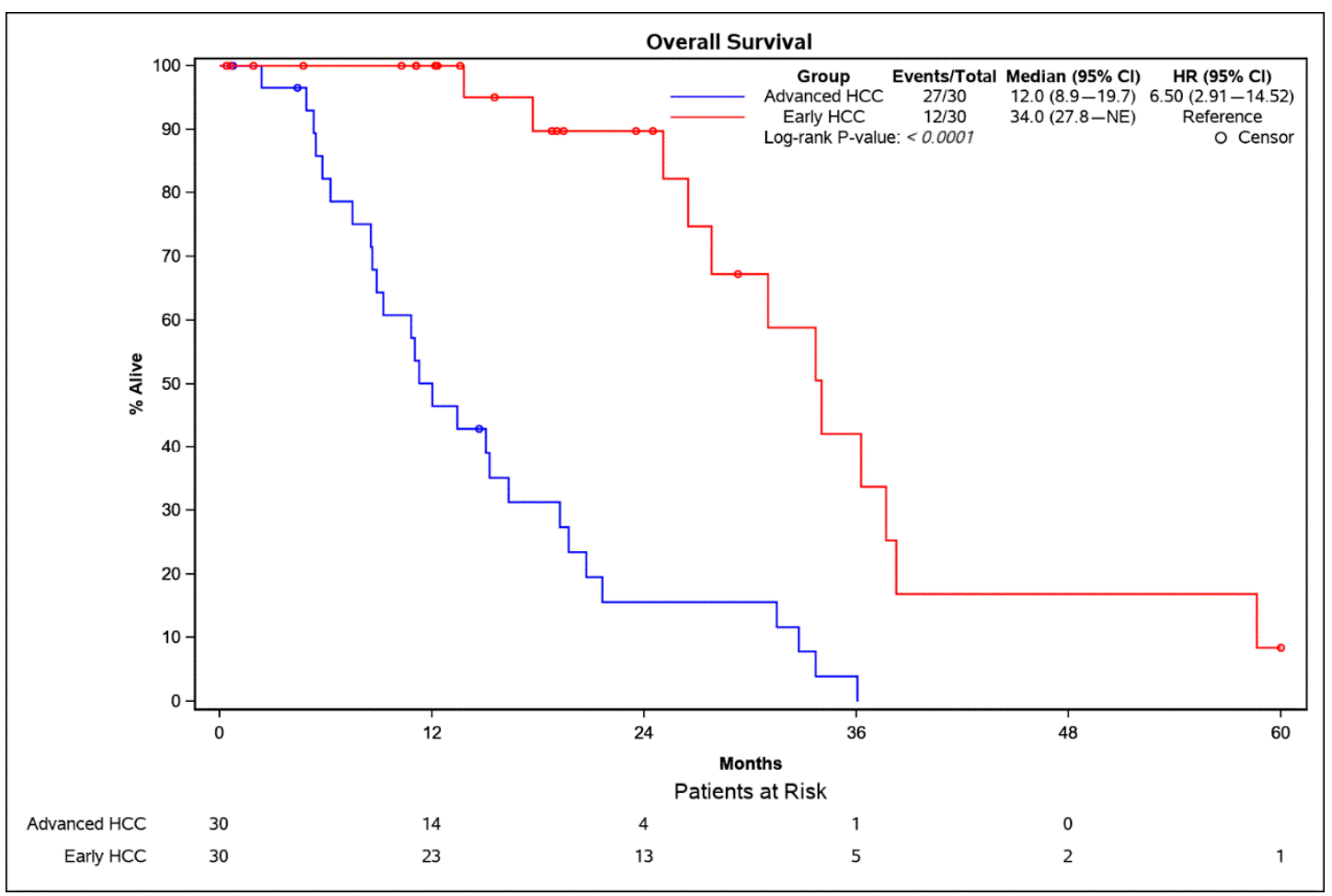

Figure 2. Kaplan-Meier survival analysis of patients with an early HCC (red) and an advanced HCC (blue). The $p$-value was calculated using the log-rank test. $\mathrm{CI}$ = confidence interval; HCC = hepatocellular carcinoma.

No significant differences in the overall survival between the different treatment groups with an early HCC (with sorafenib: 34.0 months median OS; without sorafenib: 33.7 months median OS; $p=0.4551$ ) and an advanced HCC (with SIRT: 10.4 months median OS; without SIRT: 13.4 months median OS; $p=0.7930$ ) were detected. Kaplan-Meier curves for the different treatment groups are displayed in Supplementary Figures S1 and S2.

Pearson and Spearman correlations of all tested parameters in patients with an early and an advanced HCC $(n=60)$ to the overall survival are displayed in Table 5.

The overall survival in patients with an early and an advanced HCC was compared using Kaplan-Meier analyses. The optimum cut-off values for distinguishing between low and high serum concentrations were determined using the Youden index. High serum concentrations of dimethylamine (Figure 3a) and myo-inositol (MI) (Figure 3b) were positively correlated with a higher overall survival with Pearson correlation coefficients of $0.279(p=0.034)$ and $0.331(p=0.011)$, respectively (Table 4$)$. No further parameters were positively correlated with a higher overall survival. 
Table 5. Pearson and Spearman correlations of all tested parameters in HCC patients $(n=60)$ to the overall survival. Significant $p$-values are highlighted in green. Positive correlations are highlighted in blue, negative correlations in red.

\begin{tabular}{|c|c|c|c|c|}
\hline \multirow{3}{*}{ Early and Advanced HCC } & \multicolumn{4}{|c|}{ Correlation of Parameters with Overall Survival (OS) } \\
\hline & \multicolumn{2}{|c|}{ Pearson Correlation } & \multicolumn{2}{|c|}{ Spearman Correlation } \\
\hline & $\begin{array}{l}\text { Correlation } \\
\text { Coefficient } r\end{array}$ & $p$-Value & $\begin{array}{l}\text { Correlation } \\
\text { Coefficient } r\end{array}$ & $p$-Value \\
\hline Creatine & -0.00129 & 0.9924 & -0.04675 & 0.7299 \\
\hline Creatinine & 0.14623 & 0.2777 & 0.23309 & 0.0810 \\
\hline Dimethylamine & 0.27911 & 0.0339 & 0.25011 & 0.0583 \\
\hline Dimethylsulfone & 0.17080 & 0.1999 & 0.17478 & 0.1894 \\
\hline Glycerol & -0.02346 & 0.8612 & 0.00386 & 0.9770 \\
\hline Isoleucine & 0.04831 & 0.7187 & 0.13595 & 0.3089 \\
\hline Myo-Inositol & 0.33071 & 0.0112 & 0.10792 & 0.4200 \\
\hline Valine & 0.00968 & 0.9425 & 0.14008 & 0.2943 \\
\hline GFR(NMR) & -0.20704 & 0.1223 & -0.24733 & 0.0636 \\
\hline LVLDL-P & 0.00781 & 0.9532 & 0.06300 & 0.6355 \\
\hline LDL-P & -0.36050 & 0.0050 & -0.32044 & 0.0134 \\
\hline LLDL-P & -0.33219 & 0.0102 & -0.28946 & 0.0262 \\
\hline SLDL-P & -0.31139 & 0.0164 & -0.29027 & 0.0257 \\
\hline HDL-P & 0.02843 & 0.8308 & 0.11318 & 0.3934 \\
\hline LHDL-P & -0.04053 & 0.7605 & 0.03888 & 0.7700 \\
\hline SHDL-P & -0.04230 & 0.7569 & 0.02053 & 0.8806 \\
\hline VLDL-s & -0.18036 & 0.1716 & -0.13655 & 0.3024 \\
\hline LDL-s & -0.16874 & 0.2014 & -0.11896 & 0.3695 \\
\hline HDL-s & -0.03146 & 0.8130 & 0.02049 & 0.8776 \\
\hline VLDL-c & -0.23989 & 0.0672 & -0.12459 & 0.3471 \\
\hline IDL-c & -0.28479 & 0.0288 & -0.26278 & 0.0444 \\
\hline LDL-c & -0.30483 & 0.0224 & -0.27454 & 0.0406 \\
\hline LDL.A-c & -0.32746 & 0.0114 & -0.28946 & 0.0262 \\
\hline LDL.B-c & -0.35541 & 0.0072 & -0.30513 & 0.0222 \\
\hline LDL.C-c & -0.07026 & 0.5969 & -0.03362 & 0.8005 \\
\hline HDL.A.c & -0.33777 & 0.0089 & -0.29831 & 0.0217 \\
\hline HDL.B-c & -0.13888 & 0.2942 & -0.09813 & 0.4597 \\
\hline HDL.C-c & -0.00008 & 0.9995 & 0.02121 & 0.8790 \\
\hline Total-cholesterol & -0.36757 & 0.0042 & -0.32705 & 0.0115 \\
\hline LDL-cholesterol & -0.35939 & 0.0052 & -0.35565 & 0.0057 \\
\hline HDL-cholesterol & -0.08681 & 0.5132 & -0.04480 & 0.7362 \\
\hline Triglycerides & -0.05797 & 0.6627 & -0.03840 & 0.7728 \\
\hline Alanine & -0.11190 & 0.3988 & -0.07233 & 0.5861 \\
\hline Leucine & 0.05495 & 0.6931 & 0.12933 & 0.3513 \\
\hline
\end{tabular}




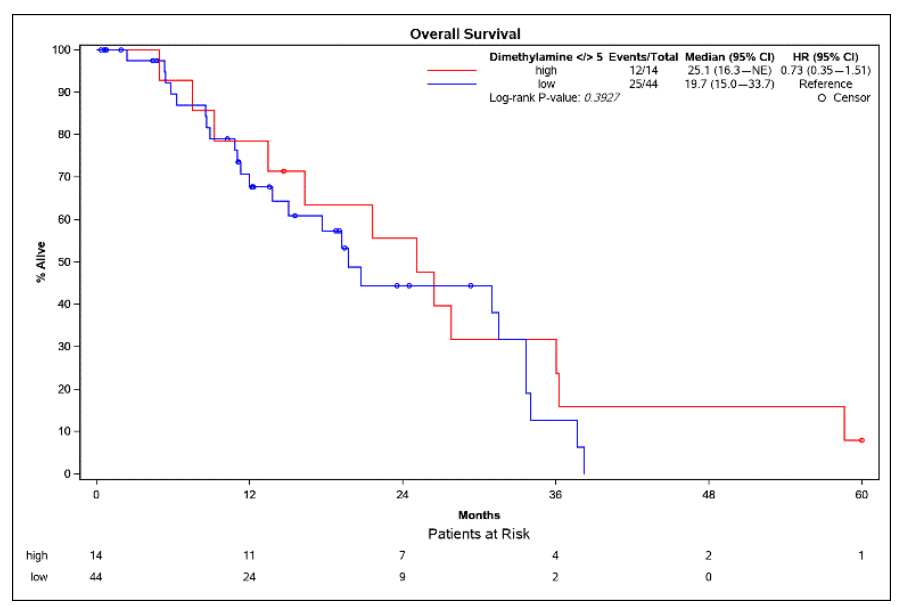

(a)

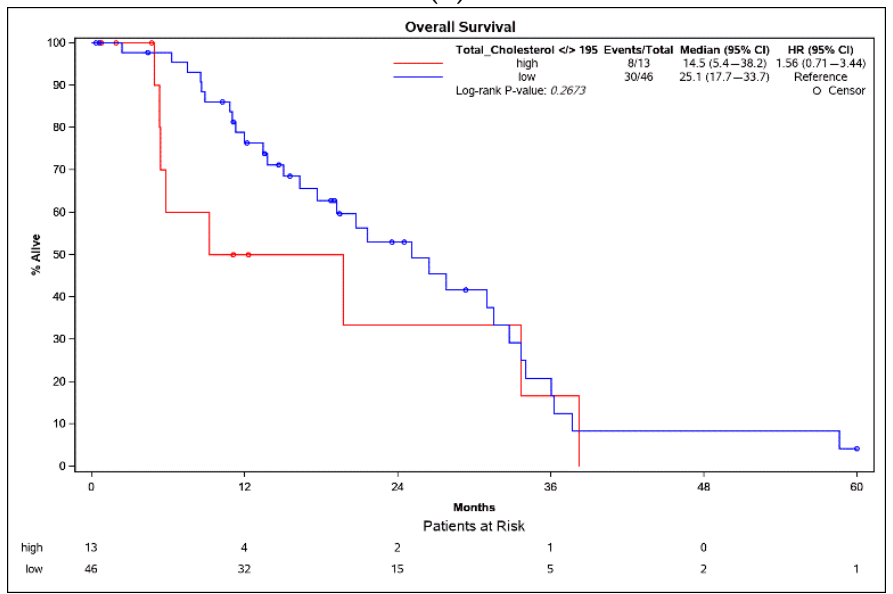

(c)

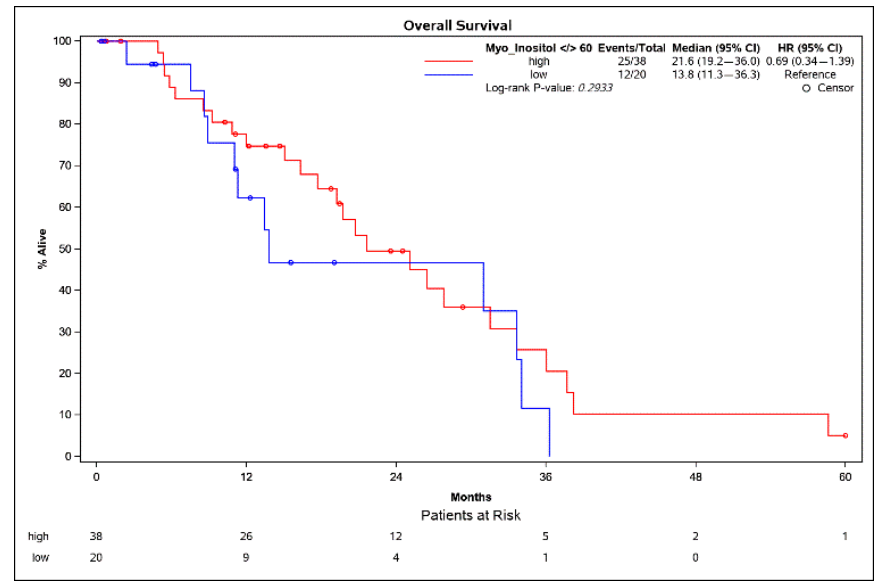

(b)

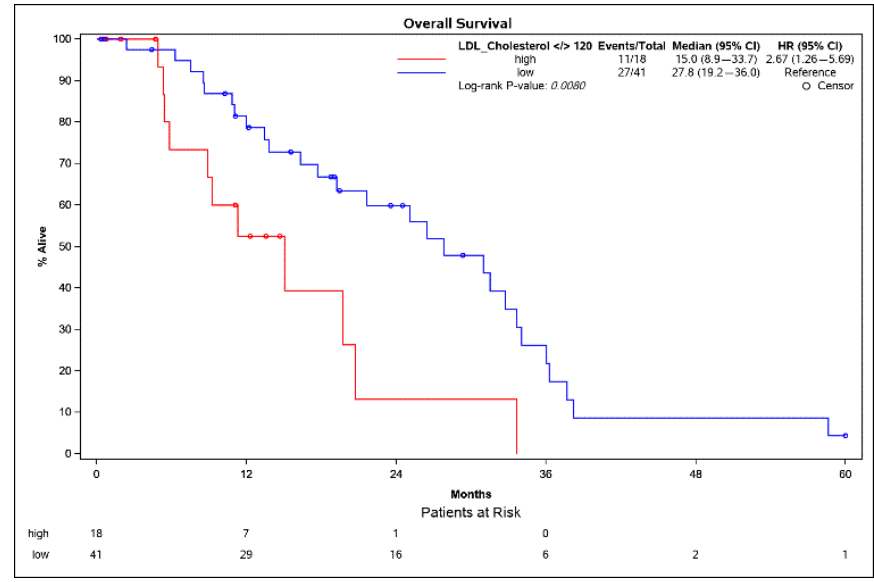

(d)

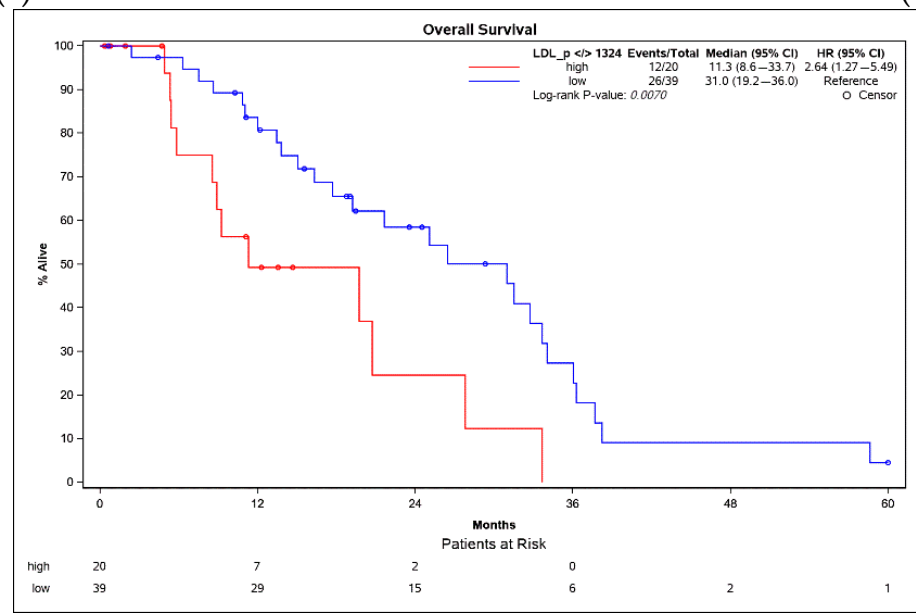

(e)

Figure 3. Kaplan-Meier survival analyses of HCC patients in correlation with the serum concentrations of dimethylamine (a), myo-inositol (b), total cholesterol (c), LDL-cholesterol (d) and LDL-P (e). The cut-off points for distinguishing between the high and low concentration values were determined by the Youden index. $p$-values were calculated using the log-rank test. $\mathrm{CI}=$ confidence interval; $\mathrm{HCC}=$ hepatocellular carcinoma. 
High serum concentrations of several parameters, however, were negatively correlated with the overall survival. The total cholesterol was negatively associated with the overall survival (Figure 3c) with a Pearson correlation coefficient of $-0.368(p=0.004)$. Patients with LDL-cholesterol above $120 \mathrm{mg} / \mathrm{dL}$ had a significantly lower overall survival (median OS: 15.0 months) compared with patients with LDL-cholesterol below $120 \mathrm{mg} / \mathrm{dL}$ (median OS: 27.8 months; $p=0.008$ ) (Figure 3d). Furthermore, the LDL particle concentration (LDL-P) was negatively associated with the overall survival with a Pearson correlation coefficient of -0.361 ( $p=0.005$ ) (Figure 3e) as were LDL subclasses LLDL-P and SLDL-P (Table 4). In addition, the cholesterol concentration in several lipoprotein subclasses (IDL-c, LDL-c, LDL.A-c, LDL.B-c and HDL.A-c) showed a significant negative correlation with overall survival.

A multivariate Cox proportional hazards model with the overall survival as the outcome variable was calculated including the parameters of patient group (early vs. advanced HCC), age ( $>$ / < 65 years), BMI (obese vs. normal and overweight vs. normal), alcohol etiology (yes vs. no), Child Pugh (A vs. B), dimethylamine (continuous), myoinositol (continuous) and LDL-P (continuous) (Table 6). In this analysis, the Cox regression showed a significant effect of the patient group $(p<0.0001)$, alcohol etiology $(p=0.0029)$, myo-inositol $(p=0.0076)$ and LDL-P $(p=0.0323)$ on the overall survival. 


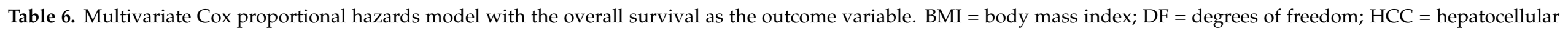
carcinoma.

\begin{tabular}{|c|c|c|c|c|c|c|c|c|c|}
\hline \multirow{2}{*}{ Parameter } & \multirow{2}{*}{ Category } & \multirow{2}{*}{ DF } & \multirow{2}{*}{$\begin{array}{l}\text { Parameter } \\
\text { Estimate }\end{array}$} & \multirow{2}{*}{$\begin{array}{l}\text { Standard } \\
\text { Error }\end{array}$} & \multirow{2}{*}{ Chi-Squared } & \multirow{2}{*}{$p$-Value } & \multirow{2}{*}{$\begin{array}{l}\text { Hazard } \\
\text { Ratio } \\
\end{array}$} & \multirow{2}{*}{\multicolumn{2}{|c|}{$\begin{array}{l}\text { 95\% Hazard Ratio } \\
\text { Confidence }\end{array}$}} \\
\hline & & & & & & & & & \\
\hline Group & Advanced HCC (vs. ref.: Early HCC) & 1 & 3.343 & 0.677 & 24.348 & $<0.0001$ & 28.296 & 7.500 & 106.746 \\
\hline Age & $\geq 65$ years (vs. ref.: $<65$ years) & 1 & 0.754 & 0.481 & 2.457 & 0.117 & 2.126 & 0.828 & 5.461 \\
\hline BMI & Obese (BMI > 30) (vs. ref.: normal) & 1 & -0.384 & 0.582 & 0.434 & 0.51 & 0,681 & 0.218 & 2.133 \\
\hline BMI & Overweight (BMI 25-30) (vs. ref.: normal) & 1 & -0.881 & 0.478 & 3.398 & 0.0653 & 0.414 & 0.162 & 1.057 \\
\hline Alcohol Etiology & Yes (vs. ref.: No) & 1 & 1.632 & 0.547 & 8.898 & 0.0029 & 5.115 & 1.750 & 14.947 \\
\hline Child Pugh & Child Pugh B (vs. ref.: Child Pugh A) & 1 & 1.023 & 0.596 & 2.949 & 0.0859 & 2.782 & 0.865 & 8.943 \\
\hline Dimethylamine & Continuous & 1 & 0.028 & 0.302 & 0.009 & 0.9264 & 1.028 & 0.569 & 1.859 \\
\hline Myo-Inositol & Continuous & 1 & -0.031 & 0.012 & 7.117 & 0.0076 & 0.969 & 0.947 & 0.992 \\
\hline LDL-P & Continuous & 1 & 0.001 & 0.000 & 4.581 & 0.0323 & 1.001 & 1.000 & 1.002 \\
\hline
\end{tabular}




\section{Discussion}

Changes in lipid profiles have been described in the presence of several types of cancer as well as various benign and malignant hepatic conditions such as hepatitis, liver cirrhosis and HCCs [19-21]. Previous studies demonstrated that altered lipid profiles occur in HCCs associated with obesity, non-alcoholic fatty liver disease (NAFLD) and a metabolic syndrome [22-24]. Furthermore, it was shown that altered lipid profiles may directly influence the tumor biology in an HCC [25-27].

This exploratory study addressed the question of whether lipid and metabolite profiles could predict the overall survival in patients with an HCC. It was shown that increased serum levels of total cholesterol and LDL-cholesterol were associated with a decreased overall survival in patients with an early and advanced HCC. In addition, several derivatives of low-density lipoprotein (LDL), which transports cholesterol from the liver to peripheral tissues, were also associated with a decreased overall survival. These findings contradicted previous studies with larger sample sizes reporting that low serum cholesterol levels were associated with poor outcomes in HCC patients undergoing curative resection. This has been suggested as being a consequence of a reduced liver functional parenchyma [28-30]. The massive destruction of liver cells in patients with an HCC leading to an increased release of cholesterol molecules might be an explanation for the negative correlation of total and LDL-cholesterol with the overall survival observed in this study. In addition, an HCC can affect the biliary tract in several ways, e.g., compression, diffuse infiltration or thrombosis [31,32]. As consecutive cholestasis is linked with increased serum levels of total cholesterol and LDL-cholesterol, higher levels may be an indicator of an elevated intrahepatic tumor load. Several studies have described that elevated serum cholesterol levels are associated with a decreased incidence of an HCC $[24,33,34]$. However, this association may be exaggerated by the fact that chronic liver diseases predisposed to the development of an HCC are linked with reduced cholesterol levels $[35,36]$. It was also shown that elevated levels of LDL particles (LDL-P) were associated with a decreased overall survival. Furthermore, this association might be explained by the known correlation between high LDL-P levels and atherosclerotic progression and the increased risk of coronary events $[37,38]$.

The results of the present study demonstrated that higher levels of serum myo-inositol (MI) were associated with a longer overall survival. MI is an isomer of inositol, formed of a six-carbon ring with each carbon hydroxylated [39]. MI has two sources: it is endogenously synthesized from glucose and it is released during digestion from dietary inositol hexaphosphate (insP6) after hydrolysis by the enzyme phytase [40,41]. MI is a component of the phospholipids located in the cellular membrane [42] and its phosphorylated derivatives cover different biological roles, insignal transduction, cellular proliferation, RNA export, DNA repair and gene expression regulation [43]. Furthermore, MI has shown chemopreventive effects in vitro and in vivo and inhibits carcinogenesis especially when in combination with insP6 [44,45]. The mechanisms triggering the anticancer activities of inositols are still not elucidated in full detail and most of the data were generated from in vitro experiments and animal models. However, clinical studies assessing the safety and chemopreventive efficacy of MI in lung cancer patients showed promising results [40,46]. Several studies have indicated that both MI and InsP6 reduce PI3K expression [47], affect EMT and cytoskeleton rearrangement [48], downregulate the Wnt pathway [49] and cause inflammation via NF-kB and TGFbeta [50] as well as modulating angiogenesis [51]. Both MI and InsP6 are strong antioxidants; however, a direct link between this property and the chemopreventive effect still needs to be demonstrated [44]. Likewise, a direct link between serum myo-inositol levels and carcinogenesis is still not clear. Interestingly, a recent meta-analysis showed that decreases in MI NMR signals in the brain correlate with the severity of hepatic encephalopathy in cirrhotic patients [52]. Although further work is needed to reveal whether this link is reflected by MI levels in the blood, this might serve as an explanation for the positive correlation of MI with the overall survival observed in this study. Further support for a role of MI in liver disease comes from a systematic review pointing towards a link between MI deficiency and NAFLD and providing evidence 
that MI supplementation has a beneficial effect in NAFLD [53], as was observed for other diseases involving insulin resistance such as polycystic ovary syndrome [54].

Furthermore, the serum concentrations of dimethylamine were associated with a higher overall survival. One main source of dimethylamine is the hydrolyzation of asymmetric dimethylarginine (ADMA), an endogenous inhibitor of nitric oxide synthase. Nitric oxide synthase releases nitric oxide from arginine, which is a potent endogenous vasodilator and is involved in the regulation of blood pressure [55]. A previous meta-analysis showed that increased ADMA as a direct precursor of dimethylamine is associated with an increased risk of coronary artery disease [56]. Another meta-analysis indicated that ADMA levels are positively associated with all-cause mortality in over 39,000 participants [57]. In light of this evidence, high levels of dimethylamine may be considered to be an indicator of proper ADMA clearance, reflecting a decreased mortality that might explain the positive correlation of dimethylamine with the overall survival in this study.

In the analyzed patient cohort, the overall survival was significantly higher in patients with an early HCC compared with patients with an advanced HCC. The NMR analysis did not reveal significant differences between the two subgroups apart from a higher cholesterol concentration in LDL subclass A in patients with an advanced HCC and a higher serum concentration of lactate in patients with an early HCC. However, the validity of this finding may be limited due to the small sample size of both subgroups. Future studies should include larger cohorts of patients with early and advanced HCCs in order to assess the potential differences between the two groups.

This study has certain limitations worth noting. First of all, this exploratory analysis focused on a relatively small sample size. The findings and hypothesis constructed require further investigations and validation in prospective studies with larger patient cohorts as a next step in developing non-invasive NMR-based metabolomic profiling as a useful diagnostic tool in HCC patients. Larger patient cohorts would also allow the exploitation of the full potential of the metabolomics approach by assessing several hundreds of metabolites and combining biomarker candidates with a significant correlation with overall survival into meaningful metabolic marker constellations. Such multimarker combinations have the potential to overcome the limitations of single markers, aiming at the most accurate prediction of therapy response and survival in HCC patients. Furthermore, the blood serum of all included patients was analyzed only once prior to therapy. Follow-up studies could focus on the evaluation of metabolic profiles during therapy in order to assess the early treatment response and evaluate potential differences between the different interventional therapies.

\section{Conclusions}

The present study demonstrated that an NMR-based assessment of lipidomic and metabolomic profiles bore the potential to identify individual metabolic biomarker candidates that could predict the outcome of patients with an HCC exposed to non-invasive therapeutic management. Follow-up studies with larger patient cohorts are needed in order to validate these results and hypotheses. Due to its reliable, easy and reproducible characterization of lipidomic and metabolomic profiles, NMR is a promising tool for developing novel biomarkers for the diagnostic and therapeutic management of HCC patients. With regard to the coming era of precision medicine and integrated diagnostics, metabolomics may become a valuable technique for predicting outcomes in HCC patients and selecting suitable therapy options tailored to individual patients in the future. The present analysis using nuclear magnetic resonance (NMR) spectroscopy showed that high serum concentrations of myo-inositol or dimethylamine were associated with an improved overall survival in HCC patients. In contrast, high concentrations of total cholesterol, LDL-cholesterol and LDL particles (LDL-P) were associated with a decreased overall survival. The identification of novel biomarkers using this NMR-based technology holds promise for opening new directions in the conduct of interventional trials in HCCs.

Supplementary Materials: The following are available online at https: / www.mdpi.com/article / 10.3390 / cancers 13112787 /s1, Figure S1. Kaplan-Meier survival analysis of patients with an early 
HCC who received treatment with sorafenib (blue) and patients with an early HCC who received treatment without sorafenib (red). The $p$-value was calculated using the log-rank test. $\mathrm{CI}=$ confidence interval; HCC = hepatocellular carcinoma, Figure S2. Kaplan-Meier survival analysis of patients with an advanced HCC who received treatment with SIRT (blue) and patients with an advanced HCC who received treatment without SIRT (red). The $p$-value was calculated using the log-rank test. CI = confidence interval; $\mathrm{HCC}=$ hepatocellular carcinoma; SIRT = selective internal radiation therapy.

Author Contributions: Conceptualization, J.R. (Jens Ricke) and P.M.; methodology, J.R. (Jens Ricke), P.M., E.S., S.H.; formal analysis, T.G.; investigation, T.G., J.R. (Johannes Rübenthaler), M.A.-F., R.S., S.W., J.M., E.S., S.H., P.M., J.R. (Jens Ricke); data curation, R.S., T.G.; writing-original draft preparation, T.G.; writing-review and editing, T.G., J.R. (Johannes Rübenthaler), M.A.-F., R.S., S.W., J.M., E.S., S.H., P.M., J.R. (Jens Ricke); visualization, R.S., T.G.; supervision, J.R. (Johannes Rübenthaler), J.R. (Jens Ricke), P.M.; project administration, J.R. (Jens Ricke), P.M. All authors have read and agreed to the published version of the manuscript.

Funding: This research received no external funding.

Institutional Review Board Statement: The study was conducted according to the guidelines of the Declaration of Helsinki and approved by all involved ethical boards. Before entering the trial, all patients gave their written informed consent (ClinicalTrials.gov No. NCT01126645; EudraCT 2009-012576-27).

Informed Consent Statement: Informed consent was obtained from all subjects involved in the study.

Data Availability Statement: The data presented in this study are available in the article and the supplementary material.

Conflicts of Interest: S.H. and E.S. are employees at Numares AG (Regensburg, Germany). All other authors declare no conflict of interest.

\section{References}

1. Wang, X.; Zhang, A.; Sun, H. Power of metabolomics in diagnosis and biomarker discovery of hepatocellular carcinoma. Hepatology 2013, 57, 2072-2077. [CrossRef] [PubMed]

2. Chaiteerakij, R.; Addissie, B.D.; Roberts, L.R. Update on biomarkers of hepatocellular carcinoma. Clin. Gastroenterol. Hepatol. 2015, 13, 237-245. [CrossRef] [PubMed]

3. Guo, W.; Tan, H.Y.; Wang, N.; Wang, X.; Feng, Y. Deciphering hepatocellular carcinoma through metabolomics: From biomarker discovery to therapy evaluation. Cancer Manag. Res. 2018, 10, 715-734. [CrossRef] [PubMed]

4. Dan, Y.; Zhang, Y.; Cheng, L.; Ma, J.; Xi, Y.; Yang, L.; Su, C.; Shao, B.; Huang, A.; Xiang, R.; et al. Hepatitis B virus X protein (HBx)-induced abnormalities of nucleic acid metabolism revealed by (1)H-NMR-based metabonomics. Sci. Rep. 2016, 6, 24430. [CrossRef] [PubMed]

5. $\quad$ Fang, M.; Dewaele, S.; Zhao, Y.P.; Stärkel, P.; Vanhooren, V.; Chen, Y.M.; Ji, X.; Luo, M.; Sun, B.M.; Horsmans, Y.; et al. Serum $\mathrm{N}$-glycome biomarker for monitoring development of DENA-induced hepatocellular carcinoma in rat. Mol. Cancer 2010, 9, 215. [CrossRef] [PubMed]

6. Suzuki, H.; Kohjima, M.; Tanaka, M.; Goya, T.; Itoh, S.; Yoshizumi, T.; Mori, M.; Tsuda, M.; Takahashi, M.; Kurokawa, M.; et al. Metabolic Alteration in Hepatocellular Carcinoma: Mechanism of Lipid Accumulation in Well-Differentiated Hepatocellular Carcinoma. Can. J. Gastroenterol. Hepatol. 2021, 2021, 8813410. [CrossRef] [PubMed]

7. Armitage, E.G.; Southam, A.D. Monitoring cancer prognosis, diagnosis and treatment efficacy using metabolomics and lipidomics. Metabolomics 2016, 12, 146. [CrossRef] [PubMed]

8. Ganti, S.; Weiss, R.H. Urine metabolomics for kidney cancer detection and biomarker discovery. Urol. Oncol. 2011, 29, 551-557. [CrossRef]

9. Stratmann, B.; Krepak, Y.; Schiffer, E.; Jarick, I.; Hauber, M.; Lee-Barkey, Y.H.; Fischer, M.; Tschoepe, D. Beneficial Metabolic Effects of Duodenal Jejunal Bypass Liner for the Treatment of Adipose Patients with Type 2 Diabetes Mellitus: Analysis of Responders and Non-Responders. Horm. Metab. Res. 2016, 48, 630-637. [CrossRef]

10. Chan, S.Y.; Capitão, L.; Probert, F.; Klinge, C.; Hoeckner, S.; Harmer, C.J.; Cowen, P.J.; Anthony, D.C.; Burnet, P.W.J. A single administration of the antibiotic, minocycline, reduces fear processing and improves implicit learning in healthy volunteers: Analysis of the serum metabolome. Transl. Psychiatry 2020, 10, 148. [CrossRef]

11. Baumstark, D.; Kremer, W.; Boettcher, A.; Schreier, C.; Sander, P.; Schmitz, G.; Kirchhoefer, R.; Huber, F.; Kalbitzer, H.R. (1)H NMR spectroscopy quantifies visibility of lipoproteins, subclasses, and lipids at varied temperatures and pressures. J. Lipid Res. 2019, 60, 1516-1534. [CrossRef] [PubMed]

12. Degoricija, V.; Potočnjak, I.; Gastrager, M.; Pregartner, G.; Berghold, A.; Scharnagl, H.; Stojakovic, T.; Tiran, B.; Marsche, G.; Frank, S. HDL subclasses and mortality in acute heart failure patients. Clin. Chim. Acta 2019, 490, 81-87. [CrossRef] [PubMed] 
13. Schettler, V.J.; Muellendorff, F.; Schettler, E.; Platzer, C.; Norkauer, S.; Julius, U.; Neumann, C.L. NMR-based lipoprotein analysis for patients with severe hypercholesterolemia undergoing lipoprotein apheresis or PCSK9-inhibitor therapy (NAPALI-Study). Ther. Apher. Dial. 2019, 23, 467-473. [CrossRef] [PubMed]

14. Banas, M.C.; Neumann, S.; Pagel, P.; Putz, F.J.; Krämer, B.K.; Böhmig, G.A.; Eiglsperger, J.; Schiffer, E.; Ruemmele, P.; Banas, B. A urinary metabolite constellation to detect acute rejection in kidney allografts. EBioMedicine 2019, 48, 505-512. [CrossRef]

15. Banas, M.; Neumann, S.; Eiglsperger, J.; Schiffer, E.; Putz, F.J.; Reichelt-Wurm, S.; Krämer, B.K.; Pagel, P.; Banas, B. Identification of a urine metabolite constellation characteristic for kidney allograft rejection. Metabolomics 2018, 14, 116. [CrossRef]

16. Ehrich, J.; Dubourg, L.; Hansson, S.; Pape, L.; Steinle, T.; Fruth, J.; Höckner, S.; Schiffer, E. Serum Myo-Inositol, Dimethyl Sulfone, and Valine in Combination with Creatinine Allow Accurate Assessment of Renal Insufficiency-A Proof of Concept. Diagnostics 2021, 11, 234. [CrossRef]

17. Ricke, J.; Klümpen, H.J.; Amthauer, H.; Bargellini, I.; Bartenstein, P.; de Toni, E.N.; Gasbarrini, A.; Pech, M.; Peck-Radosavljevic, M.; Popovič, P.; et al. Impact of combined selective internal radiation therapy and sorafenib on survival in advanced hepatocellular carcinoma. J. Hepatol. 2019, 71, 1164-1174. [CrossRef]

18. Ricke, J.; Bulla, K.; Kolligs, F.; Peck-Radosavljevic, M.; Reimer, P.; Sangro, B.; Schott, E.; Schütte, K.; Verslype, C.; Walecki, J.; et al. Safety and toxicity of radioembolization plus Sorafenib in advanced hepatocellular carcinoma: Analysis of the European multicentre trial SORAMIC. Liver Int. 2015, 35, 620-626. [CrossRef] [PubMed]

19. Jiang, J.; Nilsson-Ehle, P.; Xu, N. Influence of liver cancer on lipid and lipoprotein metabolism. Lipids Health Dis. 2006, 5, 4. [CrossRef]

20. Arain, S.Q.; Talpur, F.N.; Channa, N.A. A comparative study of serum lipid contents in pre and post IFN-alpha treated acute hepatitis C patients. Lipids Health Dis. 2015, 14, 117. [CrossRef]

21. Arain, S.Q.; Talpur, F.N.; Channa, N.A.; Ali, M.S.; Afridi, H.I. Serum lipid profile as a marker of liver impairment in hepatitis B Cirrhosis patients. Lipids Health Dis. 2017, 16, 51. [CrossRef] [PubMed]

22. Abel, S.; Smuts, C.M.; de Villiers, C.; Gelderblom, W.C. Changes in essential fatty acid patterns associated with normal liver regeneration and the progression of hepatocyte nodules in rat hepatocarcinogenesis. Carcinogenesis 2001, 22, 795-804. [CrossRef]

23. Beyoğlu, D.; Imbeaud, S.; Maurhofer, O.; Bioulac-Sage, P.; Zucman-Rossi, J.; Dufour, J.F.; Idle, J.R. Tissue metabolomics of hepatocellular carcinoma: Tumor energy metabolism and the role of transcriptomic classification. Hepatology 2013, 58, 229-238. [CrossRef]

24. Nderitu, P.; Bosco, C.; Garmo, H.; Holmberg, L.; Malmström, H.; Hammar, N.; Walldius, G.; Jungner, I.; Ross, P.; Van Hemelrijck, M. The association between individual metabolic syndrome components, primary liver cancer and cirrhosis: A study in the Swedish AMORIS cohort. Int. J. Cancer 2017, 141, 1148-1160. [CrossRef]

25. Lin, L.; Ding, Y.; Wang, Y.; Wang, Z.; Yin, X.; Yan, G.; Zhang, L.; Yang, P.; Shen, H. Functional lipidomics: Palmitic acid impairs hepatocellular carcinoma development by modulating membrane fluidity and glucose metabolism. Hepatology 2017, 66, 432-448. [CrossRef]

26. Sauer, L.A.; Dauchy, R.T.; Blask, D.E. Dietary linoleic acid intake controls the arterial blood plasma concentration and the rates of growth and linoleic acid uptake and metabolism in hepatoma 7288CTC in Buffalo rats. J. Nutr. 1997, 127, 1412-1421. [CrossRef]

27. Liu, Q.; Luo, Q.; Halim, A.; Song, G. Targeting lipid metabolism of cancer cells: A promising therapeutic strategy for cancer. Cancer Lett. 2017, 401,39-45. [CrossRef]

28. Wang, Q.; Lau, W.Y.; Zhang, B.; Zhang, Z.; Huang, Z.; Luo, H.; Chen, X. Preoperative total cholesterol predicts postoperative outcomes after partial hepatectomy in patients with chronic hepatitis B- or C-related hepatocellular carcinoma. Surgery 2014, 155, 263-270. [CrossRef]

29. Gao, X.H.; Zhang, S.S.; Chen, H.; Wang, Y.H.; Yuan, C.H.; Wang, F.B. Systemic Hepatic-Damage Index for Predicting the Prognosis of Hepatocellular Carcinoma after Curative Resection. Front. Physiol. 2017, 8, 480. [CrossRef] [PubMed]

30. Jiang, S.S.; Weng, D.S.; Jiang, L.; Zhang, Y.J.; Pan, K.; Pan, Q.Z.; Chen, C.L.; Zhao, J.J.; Zhang, X.F.; Zhang, H.X.; et al. The clinical significance of preoperative serum cholesterol and high-density lipoprotein-cholesterol levels in hepatocellular carcinoma. J. Cancer 2016, 7, 626-632. [CrossRef] [PubMed]

31. Qin, L.X.; Tang, Z.Y. Hepatocellular carcinoma with obstructive jaundice: Diagnosis, treatment and prognosis. World J. Gastroenterol. 2003, 9, 385-391. [CrossRef]

32. Bhattarai, S.; Graham, R.P.; Sigel, C.S.; Shi, J.; Gonzalez, R.S.; Xue, Y.; Krasinskas, A.M.; HooKim, K.; Adsay, V.; Reid, M.D. Bile duct involvement by hepatocellular carcinoma: A rare occurrence and poor prognostic indicator in bile duct brushing samples. Cancer Cytopathol. 2019, 127, 691-699. [CrossRef]

33. Strohmaier, S.; Edlinger, M.; Manjer, J.; Stocks, T.; Bjørge, T.; Borena, W.; Häggström, C.; Engeland, A.; Nagel, G.; Almquist, M.; et al. Total serum cholesterol and cancer incidence in the Metabolic syndrome and Cancer Project (Me-Can). PLoS ONE 2013, 8, e54242. [CrossRef]

34. Iso, H.; Ikeda, A.; Inoue, M.; Sato, S.; Tsugane, S. Serum cholesterol levels in relation to the incidence of cancer: The JPHC study cohorts. Int. J. Cancer 2009, 125, 2679-2686. [CrossRef] [PubMed]

35. Trompet, S.; Jukema, J.W.; Katan, M.B.; Blauw, G.J.; Sattar, N.; Buckley, B.; Caslake, M.; Ford, I.; Shepherd, J.; Westendorp, R.G.; et al. Apolipoprotein e genotype, plasma cholesterol, and cancer: A Mendelian randomization study. Am. J. Epidemiol. 2009, 170, 1415-1421. [CrossRef] [PubMed] 
36. Cicognani, C.; Malavolti, M.; Morselli-Labate, A.M.; Zamboni, L.; Sama, C.; Barbara, L. Serum lipid and lipoprotein patterns in patients with liver cirrhosis and chronic active hepatitis. Arch. Intern. Med. 1997, 157, 792-796. [CrossRef]

37. Cromwell, W.C.; Otvos, J.D.; Keyes, M.J.; Pencina, M.J.; Sullivan, L.; Vasan, R.S.; Wilson, P.W.; D’Agostino, R.B. LDL Particle Number and Risk of Future Cardiovascular Disease in the Framingham Offspring Study_Implications for LDL Management. J. Clin. Lipidol. 2007, 1, 583-592. [CrossRef] [PubMed]

38. Mora, S.; Buring, J.E.; Ridker, P.M. Discordance of low-density lipoprotein (LDL) cholesterol with alternative LDL-related measures and future coronary events. Circulation 2014, 129, 553-561. [CrossRef] [PubMed]

39. Thomas, M.P.; Mills, S.J.; Potter, B.V. The "Other" Inositols and Their Phosphates: Synthesis, Biology, and Medicine (with Recent Advances in myo-Inositol Chemistry). Angew. Chem. Int. Ed. Engl. 2016, 55, 1614-1650. [CrossRef]

40. Lam, S.; McWilliams, A.; LeRiche, J.; MacAulay, C.; Wattenberg, L.; Szabo, E. A phase I study of myo-inositol for lung cancer chemoprevention. Cancer Epidemiol. Biomark. Prev. 2006, 15, 1526-1531. [CrossRef]

41. Stentz, R.; Osborne, S.; Horn, N.; Li, A.W.; Hautefort, I.; Bongaerts, R.; Rouyer, M.; Bailey, P.; Shears, S.B.; Hemmings, A.M.; et al. A bacterial homolog of a eukaryotic inositol phosphate signaling enzyme mediates cross-kingdom dialog in the mammalian gut. Cell Rep. 2014, 6, 646-656. [CrossRef]

42. Antonsson, B. Phosphatidylinositol synthase from mammalian tissues. Biochim. Biophys. Acta 1997, 1348, 179-186. [CrossRef]

43. Alcázar-Román, A.R.; Wente, S.R. Inositol polyphosphates: A new frontier for regulating gene expression. Chromosoma 2008, 117, 1-13. [CrossRef] [PubMed]

44. Bizzarri, M.; Dinicola, S.; Bevilacqua, A.; Cucina, A. Broad Spectrum Anticancer Activity of Myo-Inositol and Inositol Hexakisphosphate. Int. J. Endocrinol. 2016, 2016, 5616807. [CrossRef] [PubMed]

45. Vucenik, I.; Shamsuddin, A.M. Cancer inhibition by inositol hexaphosphate (IP6) and inositol: From laboratory to clinic. J. Nutr. 2003, 133, 3778s-3784s. [CrossRef]

46. Lam, S.; Mandrekar, S.J.; Gesthalter, Y.; Allen Ziegler, K.L.; Seisler, D.K.; Midthun, D.E.; Mao, J.T.; Aubry, M.C.; McWilliams, A.; Sin, D.D.; et al. A Randomized Phase IIb Trial of myo-Inositol in Smokers with Bronchial Dysplasia. Cancer Prev. Res. 2016, 9, 906-914. [CrossRef]

47. Liu, G.; Song, Y.; Cui, L.; Wen, Z.; Lu, X. Inositol hexaphosphate suppresses growth and induces apoptosis in HT-29 colorectal cancer cells in culture: PI3K/Akt pathway as a potential target. Int. J. Clin. Exp. Pathol. 2015, 8, 1402-1410. [PubMed]

48. Dinicola, S.; Fabrizi, G.; Masiello, M.G.; Proietti, S.; Palombo, A.; Minini, M.; Harrath, A.H.; Alwasel, S.H.; Ricci, G.; Catizone, A.; et al. Inositol induces mesenchymal-epithelial reversion in breast cancer cells through cytoskeleton rearrangement. Exp. Cell Res. 2016, 345, 37-50. [CrossRef]

49. Shafie, N.H.; Mohd Esa, N.; Ithnin, H.; Md Akim, A.; Saad, N.; Pandurangan, A.K. Preventive inositol hexaphosphate extracted from rice bran inhibits colorectal cancer through involvement of Wnt/beta-catenin and COX-2 pathways. Biomed Res. Int. 2013, 2013, 681027. [CrossRef]

50. Di Sabatino, A.; Jackson, C.L.; Pickard, K.M.; Buckley, M.; Rovedatti, L.; Leakey, N.A.; Picariello, L.; Cazzola, P.; Monteleone, G.; Tonelli, F.; et al. Transforming growth factor beta signalling and matrix metalloproteinases in the mucosa overlying Crohn's disease strictures. Gut 2009, 58, 777-789. [CrossRef]

51. Singh, R.P.; Sharma, G.; Mallikarjuna, G.U.; Dhanalakshmi, S.; Agarwal, C.; Agarwal, R. In vivo suppression of hormonerefractory prostate cancer growth by inositol hexaphosphate: Induction of insulin-like growth factor binding protein-3 and inhibition of vascular endothelial growth factor. Clin. Cancer Res. 2004, 10, 244-250. [CrossRef] [PubMed]

52. Zeng, G.; Penninkilampi, R.; Chaganti, J.; Montagnese, S.; Brew, B.J.; Danta, M. Meta-analysis of magnetic resonance spectroscopy in the diagnosis of hepatic encephalopathy. Neurology 2020, 94, e1147-e1156. [CrossRef]

53. Pani, A.; Giossi, R.; Menichelli, D.; Fittipaldo, V.A.; Agnelli, F.; Inglese, E.; Romandini, A.; Roncato, R.; Pintaudi, B.; Del Sole, F.; et al. Inositol and Non-Alcoholic Fatty Liver Disease: A Systematic Review on Deficiencies and Supplementation. Nutrients 2020, 12, 3379. [CrossRef] [PubMed]

54. Unfer, V.; Facchinetti, F.; Orrù, B.; Giordani, B.; Nestler, J. Myo-inositol effects in women with PCOS: A meta-analysis of randomized controlled trials. Endocr. Connect. 2017, 6, 647-658. [CrossRef] [PubMed]

55. Tsikas, D. Urinary Dimethylamine (DMA) and Its Precursor Asymmetric Dimethylarginine (ADMA) in Clinical Medicine, in the Context of Nitric Oxide (NO) and Beyond. J. Clin. Med. 2020, 9, 1843. [CrossRef] [PubMed]

56. Xuan, C.; Tian, Q.W.; Li, H.; Zhang, B.B.; He, G.W.; Lun, L.M. Levels of asymmetric dimethylarginine (ADMA), an endogenous nitric oxide synthase inhibitor, and risk of coronary artery disease: A meta-analysis based on 4713 participants. Eur. J. Prev. Cardiol. 2016, 23, 502-510. [CrossRef] [PubMed]

57. Zhou, S.; Zhu, Q.; Li, X.; Chen, C.; Liu, J.; Ye, Y.; Ruan, Y.; Hei, Z. Asymmetric dimethylarginine and all-cause mortality: A systematic review and meta-analysis. Sci. Rep. 2017, 7, 44692. [CrossRef] 